\title{
Kelvin-Helmholtz instabilities and resonant flow instabilities for a coronal plume model with plasma pressure
}

\author{
J. Andries and M. Goossens
}

\author{
Centre for Plasma Astrophysics, K.U. Leuven, Celestijnenlaan 200B, 3001 Heverlee, Belgium \\ Received 16 October 2000 / Accepted 3 January 2001
}

\begin{abstract}
In this paper we continue the study of the effect of the velocity shear between the coronal plume and the interplume region on the spectrum of MHD waves trapped in the plume. In Andries et al. (2000) we have illustrated the concept of resonant flow instability of the trapped modes both in a 1-D slab model and a 1-D cylindrical model for a coronal plume in which plasma-pressure was neglected. The important result of that paper was that the threshold values of the velocity shear are significantly smaller for resonant instability than for Kelvin-Helmholtz instability to occur. The aim of this paper is to study the effect of plasma pressure on the eigenmodes of the plume. As expected we find slow waves in addition to the fast waves. Furthermore there are two different types of Kelvin-Helmholtz instability. Along with the fact that now not only Alfvén but also slow resonances can occur this all leads to a wide variety of ranges of the velocity shear for which instability can be present. Estimates of these ranges for different equilibrium quantities can be obtained without going through the elaborate numerical procedures of calculating the eigenmodes. We show that the instability that will most probably occur in coronal plumes is due to an Alfvén resonance of slow body modes. These instabilities could lead to disruption of the coronal plumes and to the mixing with interplume plasma. However we point out that there might be a strong dependence of the resonant flow instability upon the velocity profile that is to be investigated further.
\end{abstract}

Key words. MHD - waves - instabilities - Sun: oscillations - Sun: magnetic fields - Sun: corona

\section{Introduction}

Plumes are bright quasi-radial rays between one and several $R_{\odot}$ in coronal holes. Using SOHO-observations, Deforest et al. (1997) have unambiguously shown that all plumes lie over photospheric magnetic flux concentrations, although not all flux concentrations have plumes. Inside $10 R_{\odot}$ plumes flow much more slowly than interplume plasma (Wang 1994; Habbal et al. 1995; Grall et al. 1996; Corti et al. 1997; Poletto et al. 1997). Being bright in white light, plumes are denser than interplume plasma. All this suggests that plumes should be observable in the interplanetary medium. However, the high speed solar wind coming from coronal holes is remarkably smooth (Phillips et al. 1995). Therefore, plume and interplume plasma must be mixed somewhere close to the Sun.

There are probably numerous processes which could lead to plume/interplume mixing. Nevertheless, Suess (1998) states that it is easy to show that plumes are subject to MHD Kelvin-Helmholtz shear instabilities (KHI) beginning at around $10 R_{\odot}$ and that these instabilities otherwise lead to disruption of the plumes and to the mix-

Send offprint requests to: J. Andries,

e-mail: jesse.andries@wis.kuleuven.ac.be ing with interplume plasma. These KH-instabilities could be a potential source for some of the Alfvénic fluctuations observed in the solar wind. However, no published simulations or detailed evaluations explicitly address parameters appropriate for coronal plumes, so considerable analysis remains to be done with respect to this hypothesis (Suess 1998). Since these statements by Suess some preliminary work has been done on this subject. Parhi et al. (1999) included an ambient magnetic field in a jet simulation and found that while reducing the growth rate, the ambient magnetic field still allows for KHI if the jet speed is larger than the largest local magnetosonic speed.

The focus of this manuscript is on another possible process, also triggered by the velocity shear and which could be responsible for the plume/interplume mixing: the resonant flow instability (RFI) (Tirry et al. 1998). The aim is to show how a global MHD wave trapped in the plume can become overstable due to resonant coupling to localized Alfvén or cusp waves in the presence of a background velocity shear. This RFI occurs for velocity shears below the onset value for the KHI.

Hollweg et al. (1990) were the first to study the effect of velocity shear on the rate of resonant absorption of MHD waves supported by thin "surfaces" in 
an incompressible plasma. They found that the velocity shear can either increase or decrease the resonant absorption rate and that for certain values of the velocity shear the absorption rate goes to zero. In addition, they found that there can be resonances which do not absorb energy from the surface wave but rather give energy back to it, leading to instabilities, even at velocity shears, which are below the threshold for the KHI.

Ryutova (1988) has considered a closely related problem. She studied the propagation of kink waves along thin magnetic flux tubes in the presence of a homogeneous parallel flow outside of the tube. She was the first to introduce the concept of negative energy waves to solar physics and suggested that the resonant instability can be interpreted in terms of negative energy waves. However, according to Hollweg et al. (1990), her work contains an inconsistency. Ruderman \& Goossens (1995) studied the stability of an MHD tangential discontinuity in an incompressible plasma where viscosity is taken into account at one side of the discontinuity. The instability, which occurs for velocity shears smaller than the threshold value for the onset of the KHI, can be explained in terms of a negative energy wave which becomes unstable because of the presence of a dissipation mechanism (viscosity).

Csík et al. (1998) have investigated the reflection of MHD waves incident on a smooth boundary between two counterstreaming uniform media. They found that overreflection can occur due to wave resonance if the velocity shear is high enough.

To illustrate the concept of the RFI in coronal plumes we consider both a 1-D slab model and a 1-D cylindrical model for a coronal plume in which we ignore the geometric spreading of the coronal plume. Although the geometric spreading of the plume structure and the longitudinal gradients will certainly have an influence on the resonant flow instability mechanism, these features will not destroy the mechanism and should be taken into account in a next step, which is out of the scope of the present paper. Moreover the stability analysis of the 1-D models can be seen as a local stability analysis of the plume structure around a certain height.

Using the analytical solution around the resonance in resistive MHD to connect the solutions in ideal MHD to the left and to the right of the resonant layer, an eigenvalue code can be constructed. In this way the effect of the velocity shear on the damping rate can easily be investigated and it clearly shows how and when the resonant instability occurs. In the presence of a background flow it can be anticipated that the damping of the MHD mode due to resonant wave transformation is altered, since the flow does not only Doppler shift the continuum frequencies but it also affects the energy of the eigenmodes. The flow could drain energy away from the mode, which additionally increases the wave damping, but the flow could also be an energy source so that the mode gains energy and becomes overstable (see e.g. Tirry et al. 1998). This paper is concerned with the extension of the analysis in Andries et al. (2000) to plasma- $\beta \neq 0$.

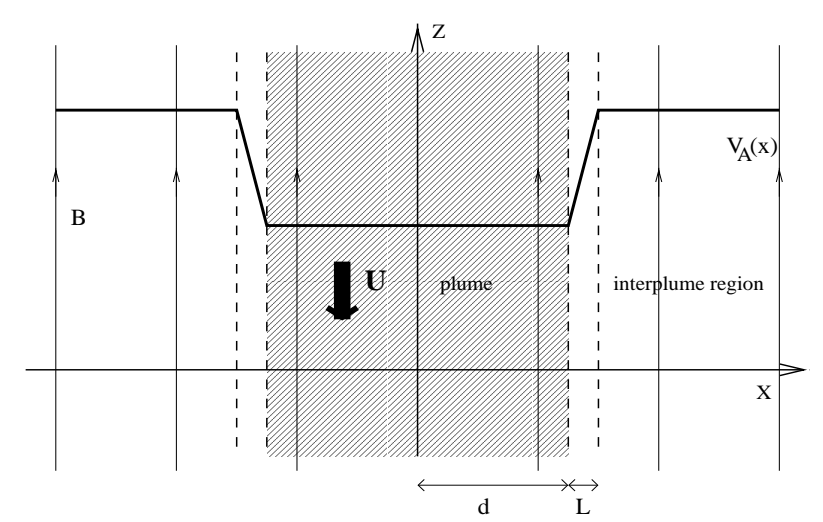

Fig. 1. A cartoon of the coronal plume model in a slab geometry

The paper is organized as follows. In Sect. 2 we describe the 1-D slab model for the coronal plume, shortly repeat the dissipative solution around the Alfvén and slow resonances. We use these solutions in the eigenvalue code to cross the singularity associated with the resonance in ideal MHD. The outcome of the eigenmode calculations is split up into two cases. First we consider a true discontinuity separating the plume and interplume regions, and show how KHI occurs by aid of the analytical dispersion relation. Then we take into account a non-uniform intermediate region which causes resonant instability. In a fourth subsection we discuss the energy exchange process. Section 3 treats the corresponding cylindrical problem. In Sect. 4 we consider the consequences of our calculations for coronal plumes. Section 5 summarizes the conclusions.

\section{Coronal plume: 1-D slab model}

\subsection{Physical model}

Figure 1 shows a cartoon of our 1-D slab model for a coronal plume in which we have ignored the geometric spreading of the plume. All equilibrium quantities depend on the $x$-coordinate only. Since in coronal holes the plasma pressure is small compared to the magnetic pressure, we have assumed $\beta$ to be zero in Andries et al. (2000). Our present goal is to investigate the influence of plasma pressure. In the model the field lines are oriented in the direction of the $z$-axis. For the computations the magnetic field is taken to be uniform over the whole space, although a different magnitude of the magnetic field in the plume and interplume region would not change the equations. The influence of such a difference (which also requires a difference in plasma-pressure, and therefore in plasma- $\beta$ ) is discussed in Sect. 4. The higher density inside the plume causes a dip in the profiles of the Alfvén and sound speed across the slab configuration. The characteristic length scale of the plume is $2 d$ whereas the thickness of the transition layer between the plume and interplume regions is $L$. In both the interior region of the plume and the exterior interplume region the Alfvén and sound speed is assumed to be uniform. In the transition layer the Alfvén speed changes 
linearly. The sound speed changes in a way so that there is pressure balance at every point in the non-uniform region. The background mass flow is smaller inside than outside the plume and changes discontinuously at $x= \pm d$. All calculations are performed in a reference frame moving with the mass flow outside the plume. Hence in this reference frame the interplume region is static whereas the plume has a background mass flow in the direction opposite to the orientation of the $z$-axis. In particular:

$U(x)=\left\{\begin{array}{ll}V, & |x|<d \\ 0, & |x| \geq d\end{array}\right.$.

Since the equilibrium quantities only depend on the $x$ coordinate, we can Fourier analyze with respect to $y$ and $z$. Hence all perturbed quantities are assumed to be proportional to:

$\exp \left[i\left(k_{y} y+k_{z} z-\omega t\right)\right]$

where $k_{y}$ and $k_{z}$ correspond to the azimuthal and the axial wave number respectively and $\omega$ denotes the frequency.

Because of the very high values of the Reynolds numbers for solar coronal conditions, dissipation due to finite electrical resisitivity and viscosity can be ignored except in narrow layers of steep gradients (e.g. around resonances). Outside these dissipative layers the MHD equations can be reduced to the following two coupled first order differential equations for the normal component of the Lagrangian displacement $\xi_{x}$ and for the Eulerian perturbation of the total pressure $P^{\prime}$ :

$D \frac{\mathrm{d} \xi_{x}}{\mathrm{~d} x}=C_{1} \xi_{x}-C_{2} P^{\prime}$

$D \frac{\mathrm{d} P^{\prime}}{\mathrm{d} x}=C_{3} \xi_{x}-C_{1} P^{\prime}$.

In absence of gravity the coefficient functions are:

$$
\begin{aligned}
D(x ; \omega) & =\rho\left(c^{2}+v_{\mathrm{A}}^{2}\right)\left(\Omega^{2}-\omega_{\mathrm{C}}^{2}\right)\left(\Omega^{2}-\omega_{\mathrm{A}}^{2}\right) \\
C_{1}(x ; \omega) & =0 \\
C_{2}(x ; \omega) & =\left(\Omega^{2}-\omega_{\mathrm{I}}^{2}\right)\left(\Omega^{2}-\omega_{\mathrm{II}}^{2}\right) \\
C_{3}(x ; \omega) & =D \rho\left(\Omega^{2}-\omega_{\mathrm{A}}^{2}\right)
\end{aligned}
$$

with:

$$
\begin{aligned}
\omega_{\mathrm{I}, \mathrm{II}}=\frac{1}{2}( & \left.k_{y}^{2}+k_{z}^{2}\right)\left(c^{2}+v_{\mathrm{A}}^{2}\right) \\
& \times\left(1 \mp\left[1-\frac{4 \omega_{\mathrm{C}}^{2}}{\left(k_{y}^{2}+k_{z}^{2}\right)\left(c^{2}+v_{\mathrm{A}}^{2}\right)}\right]^{\frac{1}{2}}\right) .
\end{aligned}
$$

The other perturbed quantities $\left(\rho_{1}, p_{1}, \ldots\right)$ can be computed once $\xi_{x}$ and $P^{\prime}$ are known. The sound speed and the Alfvén speed are defined as $c^{2}=(\gamma p) / \rho$ and $v_{\mathrm{A}}^{2}=$ $B^{2} /\left(\mu_{0} \rho\right)$ where the ratio of specific heats $\gamma=5 / 3$, as usual. The Alfvén and cusp frequency are denoted respectively by:

$$
\begin{aligned}
& \omega_{\mathrm{A}}=k_{z} v_{\mathrm{A}} \quad \text { and } \quad \omega_{\mathrm{C}}=\sqrt{c^{2} /\left(c^{2}+v_{\mathrm{A}}^{2}\right)} \omega_{\mathrm{A}} \\
& \Omega=\omega-k_{z} U(x)
\end{aligned}
$$

is the Doppler shifted frequency.

The set of ordinary differential Eqs. (1-2) has mobile regular singularities at the positions $x_{\mathrm{A}}$ and/or $x_{\mathrm{C}}$ where $D(x)$ vanishes:

$\omega=k_{z} U(x) \pm \omega_{\mathrm{A}}\left(x_{\mathrm{A}}\right)$ and/or $\omega=k_{z} U(x) \pm \omega_{\mathrm{C}}\left(x_{\mathrm{C}}\right)$. (3)

As both $\omega_{\mathrm{A}}(x)$ and $\omega_{\mathrm{C}}(x)$ are functions of $x$, they define two continuous ranges of frequencies referred to as the Alfvén continuum and the slow or cusp continuum respectively. These continua are now Doppler shifted due to the presence of the flow as indicated by Eq. (3). In the reference frame moving with the background flow in the interplume region and in the transition layer the continua are not Doppler shifted. The presence of plasma pressure in the equilibrium model, introduces the slow mode continuous spectrum besides the Alfvén spectrum and modifies the spectrum of trapped waves but the mechanism of the resonant flow instability remains the same as discussed in Andries et al. (2000).

From a physical point of view, the conditions (3) mean that the eigenmodes resonantly interact with a localised wave in one of the two continua and also with the flow. An enlightning discussion of the mechanisms by which energy is exchanged between MHD waves and a streaming background plasma is given by Walker (2000) and will be discussed in more detail in Sect. 2.4. In the absence of flow the interaction with localised waves in the continua causes damping of the eigenmodes due to resonant wave transformation and we talk about quasi-modes with complex eigenfrequency $\omega=\omega_{\mathrm{r}}+i \omega_{i}$ with $\omega_{i}<0$ in this case (Tirry $\&$ Goossens 1996 and references therein). A flow does not only cause a Doppler shift of the continuum frequencies but it can also affect the energy of the eigenmodes. The flow can namely drain energy away from the modes, which additionally increases the wave damping, but it can also be an energy source in which case the modes gain energy and become overstable, i.e. $\omega_{i}>0$.

In ideal MHD, the solutions diverge at the resonance points defined by (3). These singular solutions are nothing spectacular but just tell us that a basic ingredient is missing in the mathematical formulation of the problem. In reality, dissipation is important in a narrow layer embracing the resonant magnetic surface (Sakurai et al. 1991; Goossens et al. 1995). To remove the singularity from the ideal MHD Eqs. (1-2) we include the resistive terms into the equations for regions close to the resonances. For very high Reynolds numbers, these resistive equations represent a singular perturbation problem which can be solved analytically in the dissipative layer and in two overlap regions (to the left and the right of the dissipative layer), where ideal MHD is valid too (see e.g. Erdélyi et al. 1995; Goossens et al. 1995; Tirry et al. 1998). The dissipative solution around the Alfvén resonance $x=x_{\mathrm{A}}$ is given by (see also Tirry \& Goossens 1996):

$\xi_{x}(\tau)=-\left[\delta_{\mathrm{A}} \tau+\frac{k_{y}^{2} G_{\mathrm{A}}(\tau)}{\rho \Delta_{\mathrm{A}}}\right] C_{\mathrm{A}}+\mathcal{K}_{\mathrm{A}}$

$P(\tau)=C_{\mathrm{A}}$ 
where $C_{\mathrm{A}}$ and $\mathcal{K}_{\mathrm{A}}$ are constants of integration, $\Delta_{\mathrm{A}} \equiv$ $(\mathrm{d} / \mathrm{d} x)\left(\Omega^{2}-\omega_{\mathrm{A}}^{2}\right) . \delta_{\mathrm{A}}=\left(\omega_{\mathrm{A}} \eta /\left(\left|\Delta_{\mathrm{A}}\right|\right)\right)^{\frac{1}{3}}$ defines the length scale of the resonance layer, with $\eta$ the magnetic diffusivity. $\tau=\left(x-x_{\mathrm{A}}\right) / \delta_{\mathrm{A}}$ is a new scaled variable, which is of the order 1 in the dissipative layer. The function $G_{\mathrm{A}}(\tau)$ is given by

$G_{\mathrm{A}}(\tau)=\int_{0}^{\infty}\left[\mathrm{e}^{i u \operatorname{sign}\left(\Delta_{\mathrm{A}} \Omega_{\mathrm{r}}\right) \tau-\Lambda_{\mathrm{A}} u}-1\right] \mathrm{e}^{-u^{3} / 3} \frac{\mathrm{d} u}{u}$

where $\Lambda_{\mathrm{A}}=2 \omega_{\mathrm{A}} \omega_{i} /\left(\delta_{\mathrm{A}}\left|\Delta_{\mathrm{A}}\right|\right)$ and $\Omega_{\mathrm{r}}=\omega_{\mathrm{r}}-k_{z} U\left(x_{\mathrm{A}}\right)=$ $\pm \omega_{\mathrm{A}}$. The solution around the cusp resonance is given by:

$\xi_{x}(\tau)=-\frac{\omega_{\mathrm{C}}^{4} G_{\mathrm{C}}(\tau)}{\rho v_{\mathrm{A}}^{2} \omega_{\mathrm{A}}^{2} \Delta_{\mathrm{C}}} C_{\mathrm{C}}+\mathcal{K}_{\mathrm{C}}$

$P(\tau)=C_{\mathrm{C}}$

with $C_{\mathrm{C}}$ and $\mathcal{K}_{\mathrm{C}}$ constants of integration, $\Delta_{\mathrm{C}} \equiv$ $(\mathrm{d} / \mathrm{d} x)\left(\Omega^{2}-\omega_{\mathrm{C}}^{2}\right), \delta_{\mathrm{C}}=\left(\left(\omega_{\mathrm{C}} \eta /\left|\Delta_{\mathrm{C}}\right|\right)\left(\omega_{\mathrm{C}}^{2} / \omega_{\mathrm{A}}^{2}\right)\right)^{\frac{1}{3}}$ and now $\tau=\left(x-x_{\mathrm{C}}\right) / \delta_{\mathrm{C}}$. The function $G_{\mathrm{C}}(\tau)$ is now:

$G_{\mathrm{C}}(\tau)=\int_{0}^{\infty}\left[\mathrm{e}^{i u \operatorname{sign}\left(\Delta_{\mathrm{C}} \Omega_{\mathrm{r}}\right) \tau-\Lambda_{\mathrm{C}} u}-1\right] \mathrm{e}^{-u^{3} / 3} \frac{\mathrm{d} u}{u}$

where $\Lambda_{\mathrm{C}}=2 \omega_{\mathrm{C}} \omega_{i} /\left(\delta_{\mathrm{C}}\left|\Delta_{\mathrm{C}}\right|\right)$ and $\Omega_{\mathrm{r}}=\omega_{\mathrm{r}}-k_{z} U\left(x_{\mathrm{C}}\right)=$ $\pm \omega_{\mathrm{C}}$.

By substitution of Eq. (1) into Eq. (2) one second order differential equation in $P^{\prime}$ can be derived. For the interior of the plume and the interplume region, where all the equilibrium quantities are constant, this becomes:

$\frac{\mathrm{d}^{2} P^{\prime}}{\mathrm{d} x^{2}}=k_{x}^{2} P^{\prime}$

with:

$k_{x}^{2}=-\frac{C_{2} C_{3}}{D^{2}}=-\frac{\left(\Omega^{2}-\omega_{\mathrm{I}}^{2}\right)\left(\Omega^{2}-\omega_{\mathrm{II}}^{2}\right)}{\left(c^{2}+v_{\mathrm{A}}^{2}\right)\left(\Omega^{2}-\omega_{\mathrm{C}}^{2}\right)}$.

In a symmetric equilibrium model only symmetric and antisymmetric eigensolutions for $\xi_{x}$ and $P^{\prime}$ are possible. The antisymmetric solution for $\xi_{x}$ vanishes and changes sign when crossing $x=0$ while $P^{\prime}$ has an extremum there. This solution represents the sausage mode with the plasma being at rest along the $x=0$ plane. Similarly the symmetric solution for $\xi_{x}$ is the kink mode with the total pressure perturbation $P^{\prime}=0$ at $x=0$. The internal (indicated with subscript "i") solution for kink modes (upper sign) and sausage modes (lower sign) is:

$$
\begin{aligned}
P_{\mathrm{i}}(x) & =A\left(\mathrm{e}^{k_{x \mathrm{i}} x} \mp \mathrm{e}^{-k_{x \mathrm{i}} x}\right) \\
\xi_{x \mathrm{i}}(x) & =A \frac{k_{x \mathrm{i}}}{\rho_{\mathrm{i}}\left(\Omega_{\mathrm{i}}^{2}-\omega_{\mathrm{Ai}}^{2}\right)}\left(\mathrm{e}^{k_{x \mathrm{i}} x} \pm \mathrm{e}^{-k_{x \mathrm{i}} x}\right) .
\end{aligned}
$$

For the external region we get the general solution (indicated with subscript "e"):

$$
\begin{aligned}
P_{\mathrm{e}}(x) & =A \mathrm{e}^{k_{x \mathrm{e}} x} \\
\xi_{x \mathrm{e}}(x) & =A \frac{k_{x \mathrm{e}}}{\rho_{\mathrm{e}}\left(\Omega_{\mathrm{e}}^{2}-\omega_{\mathrm{Ae}}^{2}\right)} \mathrm{e}^{k_{x \mathrm{e}} x} .
\end{aligned}
$$

In the case where $k_{x}^{2}>0$ the solution has to vanish at infinity. Otherwise we have to impose as boundary condition that the waves are outgoing, i.e. carrying energy away from the plume (Sommerfeld condition). This depends on the sign of $\operatorname{Im}\left(k_{x}\right)$. However the choice of the root is not as straightforward as it was presented by Andries et al. (2000). In that paper we distinguished leaky modes (externally propagating modes) from trapped modes (externally evanescent modes). Modes with real frequencies in the external cut-off regions $\left[\omega_{\mathrm{ce}}, \omega_{\mathrm{Ie}}\right],\left[\omega_{\mathrm{IIe}},+\infty\right]$ (further referred to as slow and fast cut-off regions) were called leaky because $k_{x}^{2}<0$, and therefore $k_{x}$ is imaginary. It was argued that the leaky modes are carrying energy away and therefore have to be damped. But in that case $k_{x}^{2}$ is complex and the real cut-off frequencies lose their meaning. In fact damped or amplified waves are always leaky in the sense that they are carrying energy (away from the plume, as imposed). We conclude that a distinction between leaky and non-leaky modes by aid of these real cut-off frequencies is inappropriate for a description of damped or amplified modes. However this discussion makes it clear that there can be no real frequency modes within the cut-off regions. We will see that these regions lead to KHI of the eigenmodes when the real part of the frequency is forced into this regions due to the Doppler shift.

Leaving the leaky and non-leaky picture behind we return to the choice of the root of $k_{x}^{2}$. In Andries et al. (2000) we took in the cut-off region the root for which the $x$-component of the phase velocity was positive $v_{\mathrm{p}}=$ $\frac{\omega}{\operatorname{Im}\left(k_{x}\right)}>0$. This produced correct results because there was no plasma-pressure and therefore there are only fast waves in the external region. However in the presence of both magnetic field and plasma-pressure there are also slow waves for which this condition is violated while the waves are propagating outwards, since group and phase velocity point in opposite directions. This is shown in Csík et al. (1998). This would imply an evaluation of expression (17) in Csík et al. (1998) to determine which choice of the root is appropriate. We can argue however that outgoing modes that are growing will vanish at infinity since the amplitudes at infinity correspond to earlier time. Similarly outgoing modes which are damped will become unbounded at infinity. We choose the root so that $R e\left(k_{x}\right)<0$ and thereby overlook the damped outgoing waves (in which we are not interested) but we are sure to get all the amplified outgoing modes we are interested in. In addition we find some damped incoming modes which in principle do not satisfy the boundary conditions. This choice of the root is in fact the same choice as in Andries et al. (2000) for modes below the cut-off frequencies.

The procedure for solving the eigenvalue problem for the plume oscillations in our slab model is a shooting method from $x=d$ to $x=d+L$. Starting from the solution in the interior of the plume at $x=d$, we numerically integrate the ideal MHD Eqs. (1-2) towards $x=d+L$ using a fourth order Runge-Kutta method. If a resonance is encountered during the calculation, then the dissipative solutions (4) or (5) are applied continuously between the end points $x_{\mathrm{A}} \pm 6 \delta_{\mathrm{A}}$ or $x_{\mathrm{C}} \pm 6 \delta_{\mathrm{C}}$ of the corresponding dissipative layer. From Fig. 1 in Tirry \& Goossens (1996) it 
can be seen that the specific oscillatory behaviour around the resonance point caused by the effect of resistivity, indeed does not appear beyond $x_{\mathrm{A}, \mathrm{C}} \pm 6 \delta_{\mathrm{A}, \mathrm{C}}$. After having crossed the dissipative layer the computation returns to the ideal Eqs. (1-2) until the final point $x=d+L$ is reached. Application of the continuity conditions for $\xi_{x}$ and $P^{\prime}$ with the external solution at $z=d+L$ finally yields the dispersion relation which has to be solved for $\omega$ with prescribed $k_{y}, k_{z}$ and $V$.

In the calculations, length, speed, density and magnetic field strength are nondimensional and scaled with respect to $d, v_{\mathrm{Ae}}, \rho_{\mathrm{e}}$ and $B$ respectively. When $L$ is different from zero, it is taken to be $0.1 \mathrm{~d}$. In the presentation of the results $\theta$ is the angle of propagation with respect to the magnetic field, i.e. $k_{z}=k \cos (\theta)$ and $k_{y}=k \sin (\theta)$. To illustrate the concept of the resonant flow instability, it is sufficient to present only the results for the kink modes. First we take a look at the existing modes for a true discontinuity $(L=0)$.

\subsection{The eigenmodes for a true discontinuity $L=0$}

In this subsection we present the spectrum of the MHD kink modes of the slab configuration as function of the velocity shear in mass flow between the plume and interplume region. We assume that there is no transition layer. Since the interface is a true discontinuity, there is no Alfvén or cusp continuum so that the modes cannot be subject to resonant absorption. Any change in the eigenfrequency is due to the presence of the velocity shear.

We can now write down the dispersion relation analytically:

$F_{\mathrm{i}}(\omega)=F_{\mathrm{e}}(\omega)$

with:

$$
\begin{aligned}
& F_{\mathrm{i}}=\frac{P_{\mathrm{i}}^{\prime}}{\xi_{\mathrm{i}}}=\frac{\rho_{\mathrm{i}}\left(\Omega_{\mathrm{i}}^{2}-\omega_{\mathrm{Ai}}^{2}\right)}{k_{x \mathrm{i}}} \tanh \left(k_{x \mathrm{i}}\right) \\
& F_{\mathrm{e}}=\frac{P_{\mathrm{e}}^{\prime}}{\xi_{\mathrm{e}}}=\frac{\rho_{\mathrm{e}}\left(\Omega_{\mathrm{e}}^{2}-\omega_{\mathrm{Ae}}^{2}\right)}{k_{x \mathrm{e}}}
\end{aligned}
$$

Let us first look at solutions with real eigenfrequencies. In that case $F_{\mathrm{i}}$ is real whereas $F_{\mathrm{e}}$ is purely imaginary when the frequency lies within the cut-off regions and real otherwise. Therefore we conclude that there are indeed no solutions to the dispersion relation for real frequencies in the external cut-off regions. In Fig. $2 F_{\mathrm{i}}$ and $\operatorname{Re}\left(F_{\mathrm{e}}\right)$ are plotted as function of a real frequency, with the following parameters: $\theta=45^{\circ}, k=5$, density $\rho_{\mathrm{i}}=2, \beta=0.6$ and $V=0$. We can see very clearly that $F_{\mathrm{i}}$ has a series of vertical asymptotes in the internal equivalent of the external cut-off regions, $\left[\omega_{\mathrm{Ci}}, \omega_{\mathrm{Ii}}\right]$ and $\left[\omega_{\mathrm{IIi}},+\infty\right]$ making $F_{\text {i }}$ range from $-\infty$ to $+\infty$ between two asymptotes and therefore making intersection with (the real part of) $F_{\mathrm{e}}$ inevitable (above $\omega_{\text {IIi }}$ only the first intersection is a solution to the dispersion relation). These intersections correspond to the slow and fast body modes trapped in the

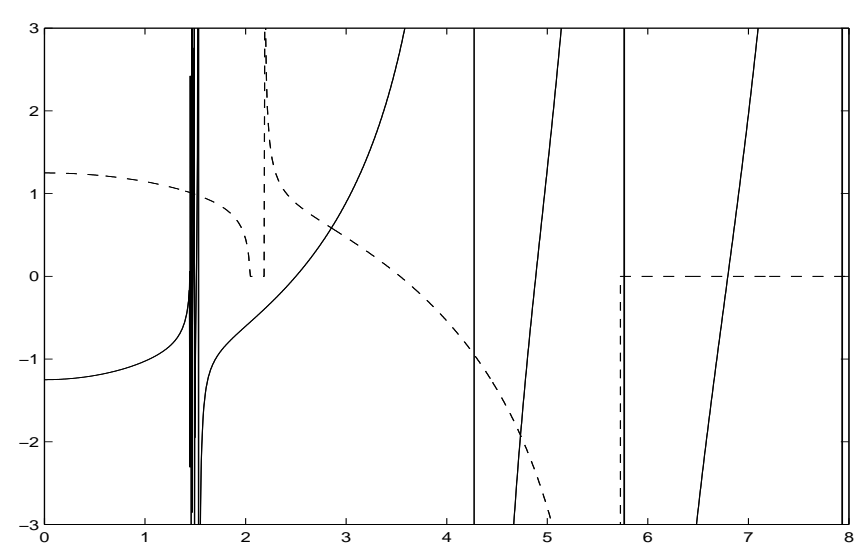

Fig. 2. The functions $\operatorname{Re}\left(F_{\mathrm{e}}\right)$ and $F_{\mathrm{i}}$ as functions of the frequency for the parameters $\theta=45^{\circ}, k=5$, density $\rho_{\mathrm{i}}=2$, $\beta=0.6$ and $V=0 . \operatorname{Re}\left(F_{\mathrm{e}}\right)$ is represented by the dashed curve, $F_{\mathrm{i}}$ by the full line. Vertical lines are asymptotes. In the regions in which $\operatorname{Re}\left(F_{\mathrm{e}}\right)=0, F_{\mathrm{e}}$ is imaginary, these are the external cut-off regions bounded by the external characteristic frequencies $\omega_{\mathrm{Ce}}=2.041, \omega_{\mathrm{Ie}}=2.185$ and $\omega_{\mathrm{IIe}}=5.721$. Intersections of the two curves (outside the cut-off regions) correspond to eigenmodes. The internal characteristic frequencies are $\omega_{\mathrm{Ci}}=1.443$, $\omega_{\mathrm{Ii}}=1.545$ and $\omega_{\mathrm{IIi}}=4.045$. Also notice that functions become zero for $\omega_{\mathrm{Ai}}=2.5$ and $\omega_{\mathrm{Ae}}=3.536$ respectively

plume structure being reflected between the two boundaries and are not present in the single boundary layer problem. We have to notice that higher order slow modes are found at lower frequencies with $\omega_{\mathrm{Ci}}$ as an accumulation point. This anti-Sturmian behaviour confirms that these are slow modes. In addition to the body modes there is an intersection in the range $\left[\omega_{\mathrm{Ii}}, \omega_{\mathrm{IIi}}\right]$, corresponding to the ordinary mode. In Andries et al. (2000) this mode was called the first fast mode although it must be distinguished from the fast and slow body modes. Whereas the body modes have imaginary $k_{x i}$ this mode has real $k_{x \mathrm{i}}$ and therefore is a surface mode. It is the only mode that is present in the single interface problem. The presence of body and surface waves in a magnetic slab is discussed by Edwin \& Roberts (1982) and in the presence of longitudinal flow by Nakariakov \& Roberts (1995) and Nakariakov et al. (1996). One can check that under these coronal conditions no surface waves are found in their papers. This is due to the fact that they only considered longitudinal waves. In that case $\omega_{\mathrm{II}}=\omega_{\mathrm{A}}$. But it is exactly in this interval $\left[\omega_{\mathrm{A}}, \omega_{\mathrm{II}}\right]$ that we find the surface waves. In the other surface wave regions $F_{\mathrm{e}}>0$ and $F_{\mathrm{i}}<0$ and thus no surface wave can be present. We come back to this point in Sect. 3.2.

In Fig. 3 we have plotted the oscillation frequency (part a) of the kink modes for the same parameters as Fig. 2 together with the imaginary part of the eigenfrequencies (part b) as function of the velocity shear $V$. The external cut-off regions are marked by the upper and lower bound of the picture for the fast region and by the horizontal dashed lines for the slow region. Only the first two slow modes are shown (very close together). The modes with positive frequency at $V=0$ are called forward 

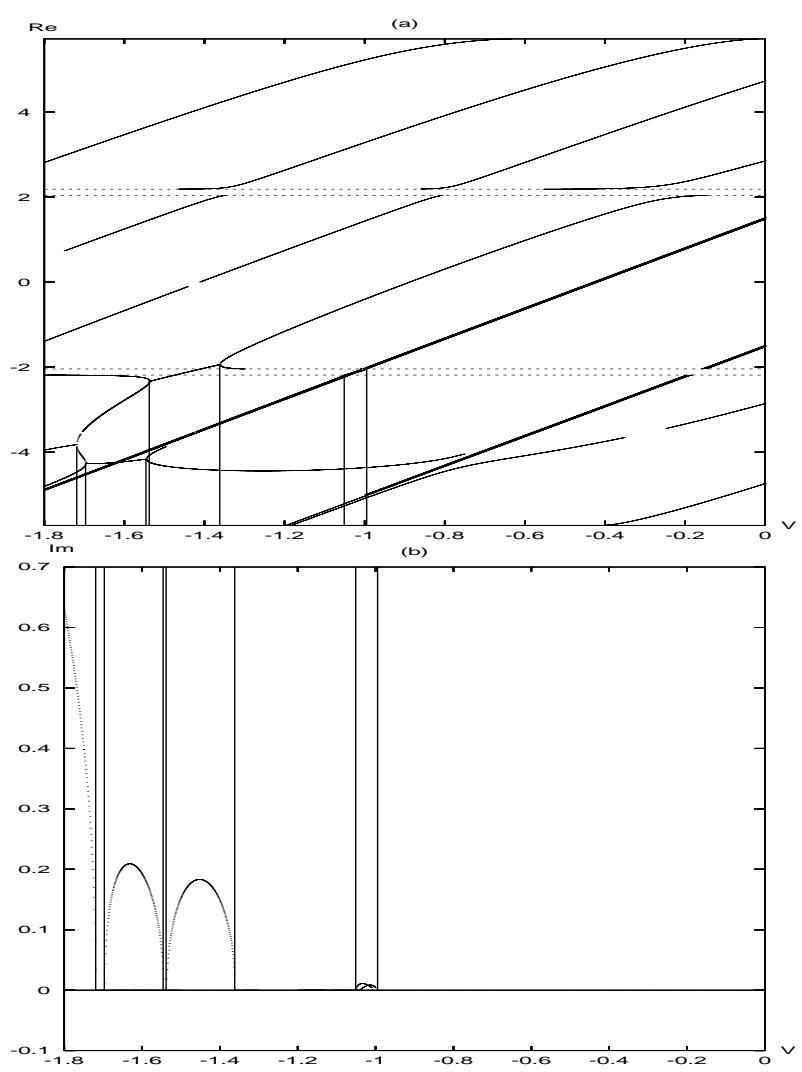

Fig. 3. a) The oscillation frequency of the kink eigenmodes as a function of the velocity shear for $L=0.1$ and the other parameters as in Fig. 2. Only the first two of the slow modes are shown very close to each other. The slow cut-off regions are marked by the horizontal dashed lines. The fast cut-off region is marked by the border of the picture. b) The corresponding imaginary part of the frequency

propagating waves (although they are shifted by the flow and may go backward with respect to the external reference frame, they are moving forward with respect to a frame fixed to the internal background flow). The modes with negative frequency at $V=0$ are called backward propagating waves. Being shifted by the flow the modes show avoided crossings and instabilities as they have to cross the external cut-off regions. The effect of the flow can be introduced into the analytical dispersion relation very easily, by noting that the only influence is a Doppler shift of the frequency inside the plume. We thus can follow these modes in their way to instability by a translation of

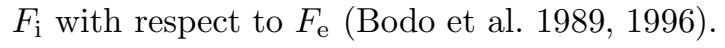

We first concentrate on the behaviour of the ordinary mode. Figure 4 a shows the avoided crossing that is needed for the forward ordinary mode to cross the slow cut-off region as it is dragged by the flow. When it approaches the backward slow cut-off region a new mode appears that merges with the ordinary mode and the curves become tangent (Fig. 4b). At this point the mode is marginally stable. At higher velocity shears the mode is destabilized, however when the velocity shear is increased further the curves become tangent again and the mode is restabilized as a new mode splits of that disappears as the velocity shear is increased further. We now look at the backward ordinary mode. Figure 4c shows the avoided crossing when the mode crosses the backward slow modes. The destabilization that occurs when crossing the forward slow modes can be visualised in a similar way as in Fig. $4 \mathrm{~b}$, but is not shown here. Finally Fig. 4d shows the destabilization due to the merging of the forward and backward ordinary mode. For higher velocity shears the ordinary mode remains unstable.

The appearance of instability of the fast and slow body waves can be explained in the same way as we have just done for the ordinary mode. Although they are fixed to their frequency range in the internal frame whereas the backward ordinary mode "traveled" between different ranges of the $F_{\mathrm{i}}$ curve (crossing the slow modes) to merge the forward ordinary mode. In case of the backward slow modes no avoided crossing is present. The modes just disappear and reappear as they cross the slow cut-off region. Similarly they disappear as they reach the fast cut-off frequency. The forward slow modes however become unstable when crossing the backward slow cut-off region and become unstable again as they enter the fast cut-off region (not shown). The fast modes behave in the same way. The vanishing of solutions and the sudden appearance of modes near the cut-off frequencies leading to instability as they merge may sound strange. In fact the solutions are found to continue as solutions that correspond to the other choice of the root of $k_{x \mathrm{e}}^{2}$ (growing incoming ones and damped outgoing ones). In general we can state that overstability occurs when forward modes meet backward external cut-off regions and that solutions avoid crossing or vanish (become damped solutions) when they enter cutoff regions of the same direction (i.e. forward/backward modes meet forward/backward cut-off regions). We conclude that there are two types of KHI, associated with the slow and the fast cut-off region respectively. We refer to them as slow and fast KHI.

The fast KHI of the ordinary mode is somewhat exceptional as it is invoked by the merging of the forward and backward ordinary modes. At zero velocity shear the ordinary modes are found near the Alfvén frequency. The influence of the strength of the magnetic field is obvious. As the internal Alfvén velocity becomes larger a higher velocity shear is needed to make the two modes merge. As in the $\beta=0$ case we find this classical KHI approximately for $|V|>v_{\mathrm{Ai}}+v_{\mathrm{Ae}}$.

It is important to make a clear distinction between the KHI of the body modes and of the ordinary (surface) modes. The KHI of the body modes clearly corresponds to the crossing of the cut-off regions and must be compared with the treatment by Ryutova (1988) of what she called "instability due to radiation of sound waves in the external medium". In our case the sound waves are replaced with fast and slow waves and hence we distinguish the fast and slow KHI. The instability of the body modes is related to the existence of propagating waves in the external medium. In our model the slow KHI of the ordinary wave is of the same kind, whereas the instability that we 

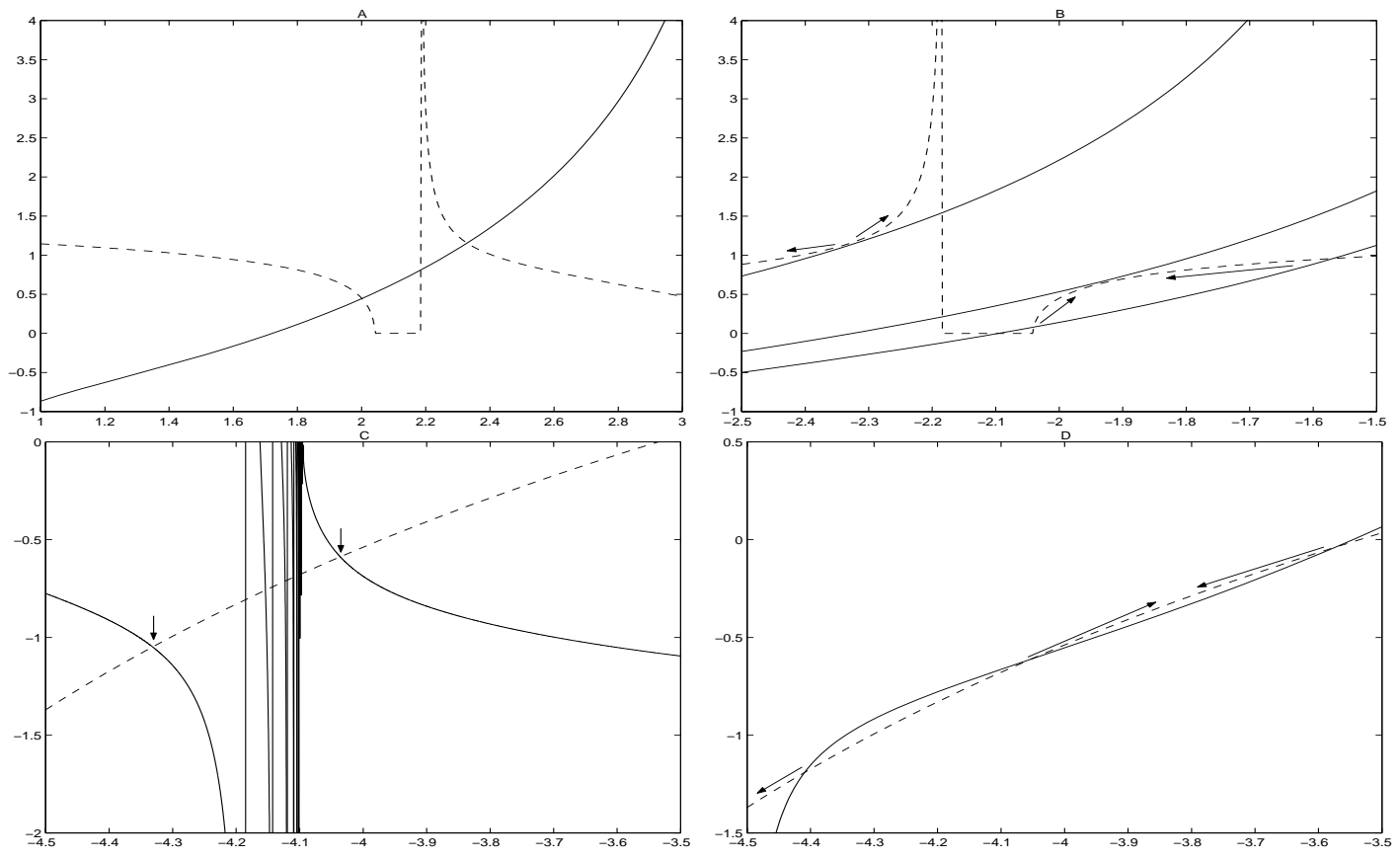

Fig. 4. Details of the functions $F_{\mathrm{i}}$ (solid) and $\operatorname{Re}\left(F_{\mathrm{e}}\right)$ (dashed). a) A detail around the slow forward cut-off region for $V=-0.3$. The two intersections correspond to the forward ordinary mode. By shifting the solid curve to the left or to the right (increasing or decreasing the value of the velocity shear) we can see that one of the two modes takes over from the other. This is the avoided crossing. b) A detail around the slow backward cut-off region. $F_{\mathrm{i}}$ (solid) is shown three times for three different values of the velocity shear $V=-1.3, V=-1.365$ and $V=-1.535$. Curves on the left correspond to higher (absolute) value of the velocity shear. The arrows indicate how the intersections move when the velocity shear is increased. c) A detail for $V=-0.8$. The intersections indicated with the arrows correspond to the two branches of the ordinary mode when it crosses the backward slow body modes (the intersections in between). d) A detail for $V=-1.7$. When the two right intersections (the forward and backward ordinary modes) merge KHI sets in. The left intersection corresponds to the mode that split of after the backward ordinary mode crossed the forward slow body modes

called the fast KHI of the ordinary wave is of another kind. It is actually the classical KHI that is also operative in an incompressible fluid (where only surface waves can be present and thus can not be caused by propagating waves in the external medium). In general the surface wave has a frequency between the inside and outside value of the Alfvén frequency at zero velocity shear. Thus in absence of a magnetic field it has a frequency equal to zero and instability is obtained for every value of the velocity shear.

\subsection{Resonant instability of the eigenmodes when $L \neq 0$}

When the true discontinuity is replaced with a nonuniform layer between the plume and interplume region, the modes with their oscillation frequency within the Alfvén or slow continuum resonantly couple to localized Alfvén or slow continuum modes, and, in absence of a velocity shear, they are damped due to the resonant wave excitation.

In Fig. $5 \mathrm{a}$ we plotted the oscillation frequencies of the ordinary and first forward and first backward slow kink modes as function of the velocity shear for the same parameters as before. In this figure we also indicate the upper and lower bounds of the slow continua $\left[-\omega_{\mathrm{Ce}},-\omega_{\mathrm{Ci}}\right]$ and $\left[\omega_{\mathrm{Ci}}, \omega_{C e}\right]$ and of the Alfvén continua $\left[-\omega_{\mathrm{Ae}},-\omega_{\mathrm{Ai}}\right]$ and
$\left[\omega_{\mathrm{Ai}}, \omega_{\mathrm{Ae}}\right]$ by the horizontal lines. These are not Doppler shifted since there is no mass flow in the nonuniform layer in our reference frame. In Fig. 5b the corresponding imaginary parts of the eigenfrequencies are plotted as function of the velocity shear $V$. In both figures we restrict the velocity shear to $-1.4<V<0$. The fast KHI and RFI for ordinary and fast waves is found for higher values of the velocity shear but is described in detail by Andries et al. (2000). We now focus on the new features arising from slow resonance and slow waves.

For $V=0$ the ordinary modes lie in the Alfvén continuum and are therefore damped. So are the slow waves due to slow resonance. As the velocity shear is increased the forward ordinary mode gets out of the Alfvén continuum and becomes stable. Subsequently it enters the slow continuum and gets damped again. In the same way the backward slow mode gets stable when it comes out of the slow continuum. When the velocity shear is increased further it becomes damped again by Alfvén resonance this time. Both slow and fast waves turn stable when they leave the corresponding continua. The interesting part appears where the forward modes get into the backward continua. In this case they are amplified as we have already shown for the Alfvén resonance of fast waves. The picture is completely the same. The slow forward mode gets amplified due to the slow resonance for 

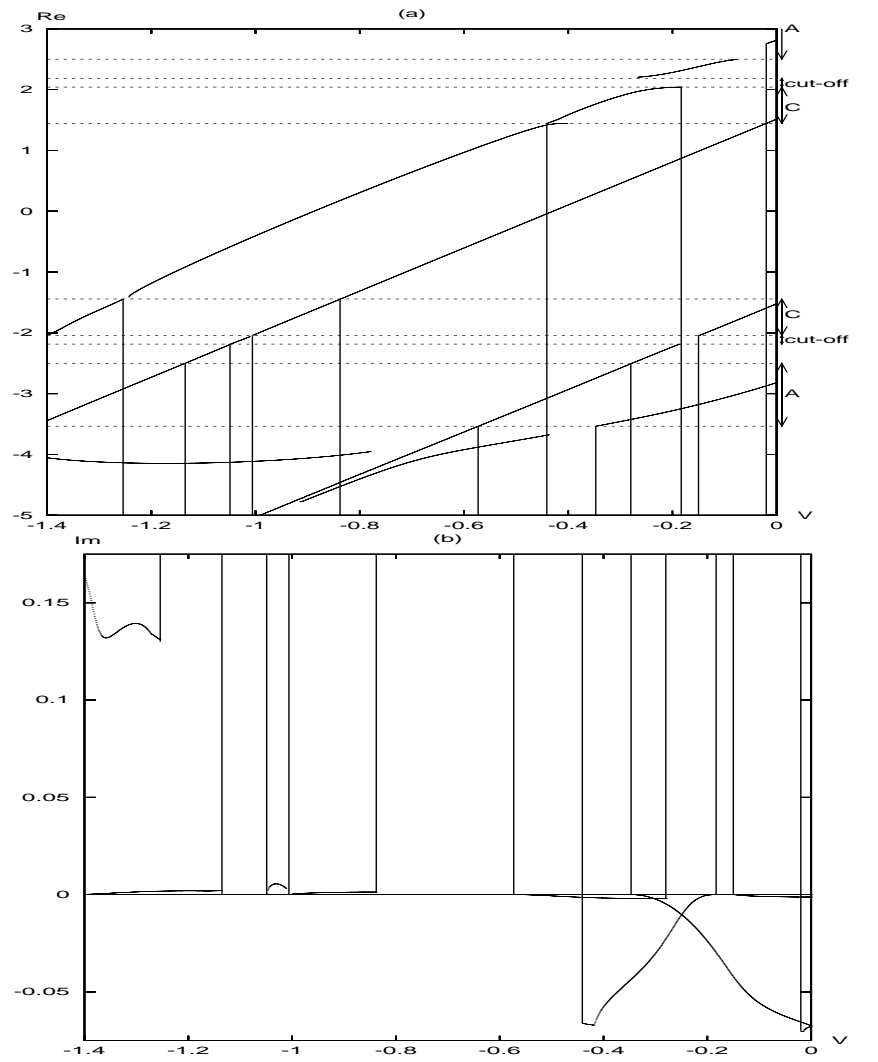

Fig. 5. a) The oscillation frequency of the first slow and the ordinary kink mode as a function of the velocity shear for $L=0.1$ and the other parameters as in Fig. 2. The continua and the slow cut-off regions are marked (A, C and cut-off respectively). The fast cut-off regions lie outside the picture. b) The corresponding imaginary part of the frequency

velocity shears $-0.84<V<-1$, and due to the Alvén resonance for $-1.42<V<-1.14$. The ordinary mode is amplified by slow resonance around $-1.4<V<-1.25$. We must emphasize here that plasma- $\beta$ is taken to be 0.6 which is to high for the low corona, and that the velocity ranges for which instability occurs are highly dependent on $\beta$. The dependence on the equilibrium parameters and its implications for the stability of plumes will be discussed in Sect. 4.

\subsection{The energy transfer}

Andries et al. (2000) have explained the resonant overstabilities using negative energy waves (the slow resonance can be treated in the same way as the Alfvén resonance). In that case the wave energy flux is defined in a way that it is continuous over the flow boundary. We calculated the flux of energy going into the resonance layer and found it to be negative when the velocity shear is sufficiently high, i.e.:

$\frac{\Omega_{\mathrm{i}}}{\Omega_{\mathrm{e}}}<0$

We adopt another approach now and use the energy and energy flux definitions by Walker (2000). In Walker's defi-

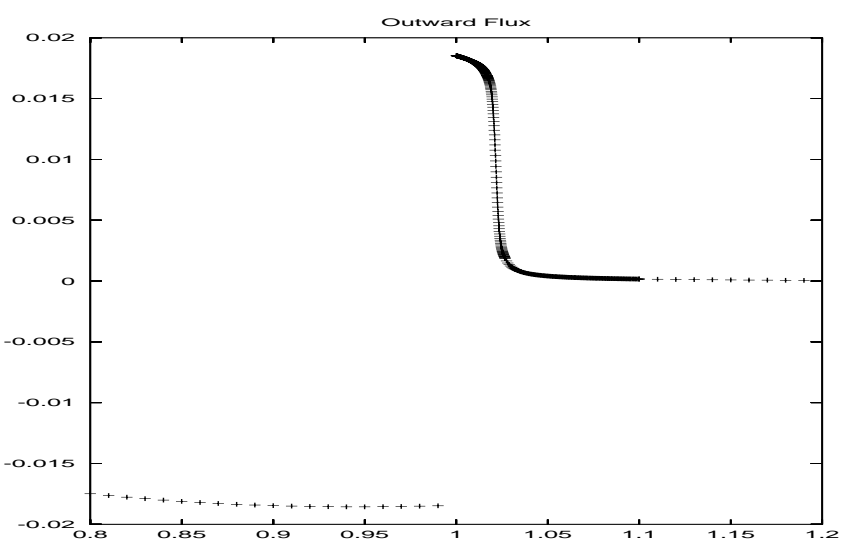

Fig. 6. The outward energy flux (as defined by Walker) as a function of position. The discontinuous flow boundary couples the outward flux to an inward flux

nition not the energy flux itself but the energy flux devided by the Doppler shifted frequency is continuous over a discontinuous flow boundary. First of all we used Walker's definition to check whether the modes are outgoing or incoming. With this definition the flux points in the direction of the group velocity, which is not the case when the negative energy wave definition is used. In fact in the negative energy wave definition the outward energy flux is different when computed in different reference frames moving relative to each other along the plume direction. We think little advantage can be found in such a viewpoint. We found that $\operatorname{Re}(\omega) / \operatorname{Im}\left(k_{x \mathrm{e}}\right)$, the phase velocity in the $x$-direction outside the plumes of modes that are unstable due to slow KHI or slow RFI is opposite to the $x$-component of the group velocity. Whereas it is parallel for fast KHI or Alfvén RFI. This is consistent with the before mentioned calculation by Csík et al. (1998) of the slow and fast characteristics of the external waves.

Using Walker's definition the energy flux into the resonance layer is positive always. Although the calculations done with the negative energy wave picture are correct, this is a good example of how it obscures the localization of the energy exchange. How can the resonance layer that transforms magnetic and kinetic energy into heat emit energy? That is indeed impossible. It is the flow that delivers the energy and the energy transfer is localized at the discontinuous flow boundary and not in the resonance layer. This is clearly shown in Fig. 6. The effect of the resonance is only to provoke energy transfer which makes the flow give energy to the wave. The outward energy flux at the outside of the boundary (the resonance sucks energy away) couples to an inward energy flux at the inside of the boundary due to the frequency and jump in the flow velocity that satisfy (7) (see Walker 2000).

In this way also the KHI can be explained. Because the external wave transports energy away for frequencies in the cut-off regions this creates an outward flux at the external side of the flow boundary. Due to the jump in the flow velocity this outward flux couples to an inward flux at the inside of the flow boundary when Eq. (7) is 
satisfied. The inward flux represents an inflow of energy that is trapped in the plume and causes the wave to grow. Whether the outward flux is created by outward propagating waves or by resonant absorption is of minor importance. However we now can see clearly that the results concerning resonant instability critically depend on the localization of the discontinuous flow boundary. This is because the velocity difference between the resonant position and the internal is crucial for the resonant instability process. The effects of different flow boundaries, including a smooth change in background flow, are to be investigated in a future paper. At this moment we can only suggest that the results are applicable if the flow boundary is very steep and localized at the inside boundary of the transition region for the density (or magnetic field). In this respect we note that in a three-dimensional simulation of a heavy magnetized jet with large Alfvénic Mach number Hardee \& Clarke (1995) found that the transverse profile of the velocity was much broader than the density and magnetic field profile. This means that in that case the velocity boundary is not at all a steep boundary located at the inside of the density (or magnetic field) transition region. And this can probably be a more stable configuration.

Our explanation in terms of the backward/forward coupling (coupling internal negative frequency waves with external positive frequency waves and vice versa) must be related to the discussion by Cairns (1979) about the role of negative energy waves in instabilities of parallel flows. Figure 3 in that paper shows exactly the features we obtain in Fig. 3 (although he uses the wave number on the $x$-axis, whereas we use the velocity shear). Cairns' explanation is in terms of the sign of the energy of the two coupled modes. Although we are not happy with the frame dependent energy and flux definitions his idea is essentially equivalent to Eq. (7). Ryutova (1988) also uses the concept of negative energy waves. To illustrate the correspondence with our backward/forward explanation, one can check the following. The threshold she obtains for the appearance of negative energy waves (7) can be substituted in her dispersion relation (4) to find that it is precisely the threshold for which the negative sign solution (externally backward) starts having a positive internal frequency. And thus our Eq. (7) is satisfied.

\section{Coronal plume: 1-D cylindrical model}

\subsection{Physical model}

In this section we consider the cylindrical equivalent of the slab configuration. All equilibrium quantities now depend on the $r$-coordinate only. The characteristic radius of the plume is $d$ whereas the thickness of the transition layer between the plume and interplume region is still $L$. Again we assume the plasma- $\beta$ parameter and the magnetic field to be constant in the entire space. The Alfvén and sound speed are uniform in the interior region of the plume and the exterior interplume region. In the transition layer the
Alfvén and sound speed change linearly. In the reference frame used in the eigenmode calculations the background mass flow is now given by:

$U(r)= \begin{cases}V, & r<d \\ 0, & r \geq d\end{cases}$

In the cylindrical coordinates all perturbed quantities are assumed to be proportional to:

$\exp \left[i\left(m \phi+k_{z} z-\omega t\right)\right]$

where $m$ is the azimuthal wave number. For a compressible $1 \mathrm{D}$ cylindrical plasma the ideal MHD equations can be reduced to a set of two first-order differential equations for the radial component of the Lagrangian displacement $\xi_{\mathrm{r}}$ and the Eulerian perturbation of the total pressure $P^{\prime}$ (see e.g. Appert et al. 1974):

$$
\begin{aligned}
& D \frac{\mathrm{d} r \xi_{\mathrm{r}}}{\mathrm{d} r}=C_{1} r \xi_{\mathrm{r}}-C_{2} r P^{\prime} \\
& D \frac{\mathrm{d} P^{\prime}}{\mathrm{d} r}=C_{3} \xi_{\mathrm{r}}-C_{1} P^{\prime}
\end{aligned}
$$

where now for the cylindrical configuration:

$$
\begin{aligned}
\omega_{\mathrm{I}, \mathrm{II}}= & \frac{1}{2}\left(\frac{m^{2}}{r^{2}}+k_{z}^{2}\right)\left(c^{2}+v_{\mathrm{A}}^{2}\right) \\
& \times\left(1 \pm\left[1-\frac{4 \omega_{\mathrm{C}}^{2}}{\left(\frac{m^{2}}{r^{2}}+k_{z}^{2}\right)\left(c^{2}+v_{\mathrm{A}}^{2}\right)}\right]^{\frac{1}{2}}\right) .
\end{aligned}
$$

As Eqs. (1-2), this set of ordinary differential equations has two mobile regular singularities at the positions $r_{\mathrm{A}}$ and/or $r_{\mathrm{C}}$ where $D(r)$ vanishes:

$\omega=k_{z} U(r) \pm \omega_{\mathrm{A}}\left(r_{\mathrm{A}}\right) \quad$ and $/$ or $\quad \omega=k_{z} U(r) \pm \omega_{\mathrm{C}}\left(r_{\mathrm{C}}\right)$.

For the present cylindrical configuration the dissipative solution around the Alfvén resonance is given by:

$\xi_{\mathrm{r}}(\tau)=-\frac{m^{2} C_{\mathrm{A}}}{r_{\mathrm{A}}^{2} \rho_{\mathrm{A}} \Delta_{\mathrm{A}}} G_{\mathrm{A}}(\tau)+\mathcal{K}_{1}$

$P(\tau)=C_{\mathrm{A}}$

where $C_{\mathrm{A}}$ and $\mathcal{K}_{1}$ are constants of integration, $\Delta_{\mathrm{A}} \equiv$ $(\mathrm{d} / \mathrm{d} r)\left(\Omega^{2}-\omega_{\mathrm{A}}^{2}\right) \cdot \delta_{\mathrm{A}}=\left(\omega_{\mathrm{A}} \eta /\left|\Delta_{\mathrm{A}}\right|\right)^{\frac{1}{3}}$ defines the length scale of the resonance layer. $\tau=\left(r-r_{\mathrm{A}}\right) / \delta_{\mathrm{A}}$ is the new scaled variable, which is of the order 1 in the dissipative layer. Around the slow resonance the dissipative solution is the same as $(5)$ with $\Delta_{\mathrm{C}} \equiv(\mathrm{d} / \mathrm{d} r)\left(\Omega^{2}-\omega_{\mathrm{C}}^{2}\right)$.

For the interior of the plume and the interplume region where all the equilibrium quantities are constant, the second order differential equation in $P^{\prime}$ now becomes Bessel's equation:

$\frac{\mathrm{d}^{2} P^{\prime}}{\mathrm{d} r^{2}}+\frac{1}{r} \frac{\mathrm{d} P^{\prime}}{\mathrm{d} r}-\left(\frac{m^{2}}{r^{2}}+k_{x}^{2}\right) P^{\prime}$

with:

$k_{x}^{2}=-\frac{C_{2}(m=0) C_{3}}{D^{2}}=-\frac{\left(\Omega^{2}-\omega_{\mathrm{A}}^{2}\right)\left(\Omega^{2}-k_{z}^{2} c^{2}\right)}{\left(c^{2}+v_{\mathrm{A}}^{2}\right)\left(\Omega^{2}-\omega_{\mathrm{C}}^{2}\right)}$. 
The internal solution, which has to satisfy the regularity condition at the axis $r=0$, is given by:

$\begin{aligned} P_{\mathrm{i}}(r) & =A I_{m}\left(k_{x \mathrm{i}} r\right) \\ \xi_{\mathrm{ri}}(r) & =A \frac{k_{x i}}{\rho_{\mathrm{i}}\left(\Omega_{\mathrm{i}}^{2}-\omega_{\mathrm{Ai}}^{2}\right)} I_{m}^{\prime}\left(k_{x \mathrm{i}} r\right) .\end{aligned}$

For the external region the solution that vanishes at infinity is:

$$
\begin{aligned}
P_{\mathrm{e}}(r) & =A K_{m}\left(k_{x \mathrm{e}} r\right) \\
\xi_{\mathrm{re}}(r) & =A \frac{k_{x e}}{\rho_{\mathrm{e}}\left(\Omega_{\mathrm{e}}^{2}-\omega_{\mathrm{Ae}}^{2}\right)} K_{m}^{\prime}\left(k_{x \mathrm{e}} r\right)
\end{aligned}
$$

with the root taken to have positive real part. $I_{m}$ and $K_{m}$ denote the modified Bessel function of the first and second kind respectively of order $m$. The prime on these symbol denotes the derivative with respect to their argument.

\subsection{The eigenmodes and instabilities}

The dispersion relation is the same as $(6)$ with $F_{\mathrm{i}}$ and $F_{\mathrm{e}}$ now given by:

$$
\begin{aligned}
& F_{\mathrm{i}}=\frac{\rho_{\mathrm{i}}\left(\Omega_{\mathrm{i}}^{2}-\omega_{\mathrm{Ai}}^{2}\right)}{k_{x \mathrm{i}}} \frac{I_{m}\left(k_{x \mathrm{i}}\right)}{I_{m}^{\prime}\left(k_{x \mathrm{i}}\right)} \\
& F_{\mathrm{e}}=\frac{\rho_{\mathrm{e}}\left(\Omega_{\mathrm{e}}^{2}-\omega_{\mathrm{Ae}}^{2}\right)}{k_{x \mathrm{e}}} \frac{K_{m}\left(k_{x \mathrm{e}}\right)}{K_{m}^{\prime}\left(k_{x \mathrm{e}}\right)} .
\end{aligned}
$$

These functions look very much the same as in the slab geometry. For real frequencies $F_{\mathrm{e}}$ takes complex values in the cut-off regions and can therefore not be equal to $F_{\mathrm{i}}$ which is real. The cut-off regions are now given by $\left[\omega_{\mathrm{Ce}}, k_{z} c_{\mathrm{e}}\right]$ and $\left[\omega_{\mathrm{Ae}},+\infty[\right.$. The fast and slow modes are found between $\left[\omega_{\mathrm{Ci}}, k_{z} c_{\mathrm{i}}\right]$ and $\left[\omega_{\mathrm{Ai}},+\infty[\right.$ respectively. We can see in Fig. 7 for parameters $\beta=0.6, \rho=2, m=1$, $k_{z}=5$ that no ordinary mode can be present here because outside the regions of the fast and slow body modes $F_{\mathrm{i}}$ is positive whereas $F_{\mathrm{e}}$ is always negative. The existence of surface and body waves in a magnetic cylinder is discussed by Edwin \& Roberts (1983). Under coronal conditions they indeed found no surface waves. As we previously mentioned under these conditions no longitudinal surface waves are found in the slab model either. But nonlongitudinal surface waves are present in the slab. In the surface wave regions the sign of the functions $F_{\mathrm{i}}$ and $F_{\mathrm{e}}$ is determined by the sign of $\Omega^{2}-\omega_{A}^{2}$ (as well in the cylindrical model as in the slab model). Therefore we can see that surface waves are to be found between the internal and external Alfvén frequency just as in the incompressible case. And therefore in our model the surface wave has a frequency above the internal Alfvén frequency. If we look at longitudinal waves the frequencies $\omega_{\mathrm{I}}$ and $\omega_{\text {II }}$ reduce to $k_{z} c$ and $\omega_{\mathrm{A}}$ just as in the cylindrical case. Thus in our low $\beta$ model waves with frequencies above the internal Alfvén frequency are body modes and no surface mode can be present. One can intuitively understand that waves in a cylinder are in a sense longitudinal since the $\theta$ domain is finite contrary to the $y$ domain in a slab geometry.

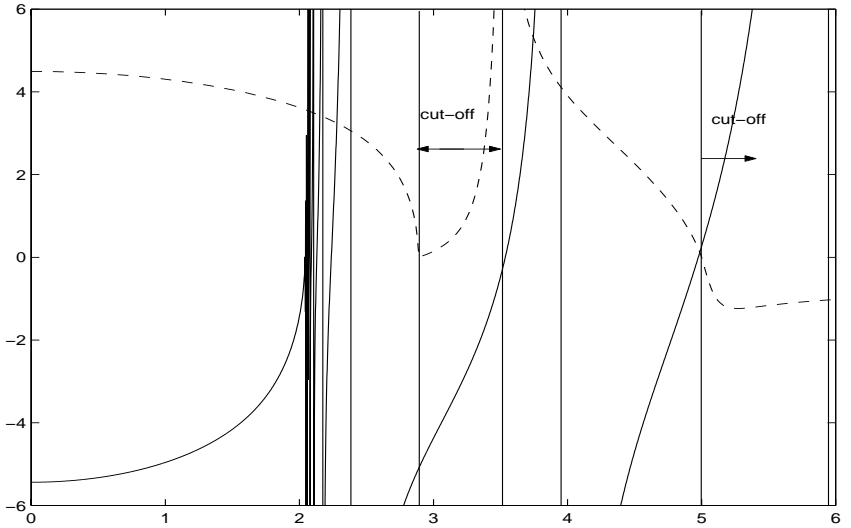

Fig. 7. The functions $\operatorname{Re}\left(F_{\mathrm{e}}\right)$ and $F_{\mathrm{i}}$ as functions of the frequency for the parameters $m=1, k_{z}=5$, density $\rho_{\mathrm{i}}=2$, $\beta=0.6$ and $V=0 . \operatorname{Re}\left(F_{\mathrm{e}}\right)$ is represented by the dashed curve, $F_{\mathrm{i}}$ by the full line. Vertical lines are asyptotes. In the marked cut-off regions $F_{\mathrm{e}}$ is complex. The cut-off frequencies are $\omega_{\mathrm{Ce}}=2.887, k_{z} c_{\mathrm{e}}=3.536$ and $\omega_{\mathrm{Ae}}=5$. Intersections of the two curves (outside the cut-off regions) correspond to eigenmodes. The internal characteristic frequencies are $\omega_{\mathrm{Ci}}=2.041$, $k_{z} c_{\mathrm{i}}=2.5$ and $\omega_{\mathrm{Ai}}=3.536$. Also notice that functions become zero for $\omega_{\mathrm{Ai}}$ and $\omega_{\mathrm{Ae}}$ respectively

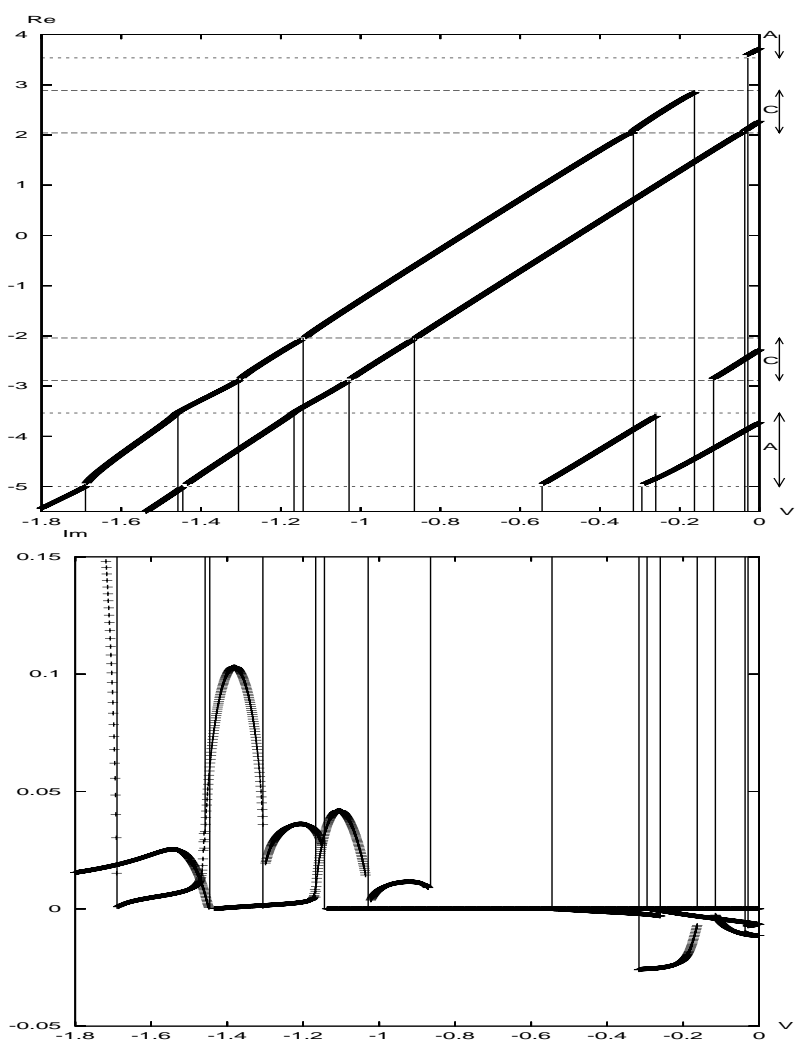

Fig. 8. a) The oscillation frequency of the first slow and first fast waves as a function of the velocity shear for $L=0.1$ and the other parameters as in Fig. 7. The continua are marked. The fast cut-off region lies above the Alfvén continuum and the slow cut-off region lies just in between the cusp (C) and Alfvén (A) continuum. It is a pure coincidence that for the parameters chosen the upper bound of the slow cut-off region is equal to the internal Alfvén frequency. b) The corresponding imaginary part of the frequency 
For the results of the eigenmode calculations we immediately turn to the case $L \neq 0$. As in the slab model KHI sets in when forward modes have to cross backward cutoff regions. Resonant instability occurs if forward modes have to cross backward continua. This can be seen in Fig. 8. Part (a) shows the oscillation frequencies of the first slow mode and the first fast mode as a function of the velocity shear for the same parameters as in Fig. 7. Part (b) shows the corresponding imaginary parts. The continua are marked. The fast cut-off region lies above the Alfvén continuum and the slow cut-off region lies just in between the cusp and Alfvén continuum. It is a pure coincidence that for the parameters chosen the upper bound of the slow cut-off region is equal to the internal Alfvén frequency. The same discussions as in the slab geometry apply here.

\section{Dependence on the parameters and application to coronal plumes}

A clear message from our discussion is that the range of frequencies for which slow and fast body modes occur can be determined by aid of the characteristic frequencies only. This is true for both slab and cylindrical configurations. For the slab configuration the ranges also depend on the angle of propagation (through $\omega_{\mathrm{I}}$ and $\omega_{\mathrm{II}}$ ). This complicates the analysis of the dependence on the parameters and there is no need to go through that trouble because the cylindrical case is without doubt better adopted to astrophysical application and does not have this complication. Furthermore if $\theta \rightarrow 0$, then $\omega_{\mathrm{I}} \rightarrow k_{z} c$ and $\omega_{\mathrm{II}} \rightarrow \omega_{\mathrm{A}}$ and thus equivalent results are obtained for the slab and cylindrical model. Therefore we further restrict our attention to the cylindrical case, thereby closing the discussion about the influence of the angle of propagation on the velocity shears for which instability occurs. The behaviour of the ordinary modes is somewhat different and less confined to its frequency range, and is even absent in our specific cylindrical case. Therefore we will only look at the body modes.

Furthermore we showed how KHI occurred by crossing the cut-off regions. RFI is present when crossing the continua. These findings make it unnecessary to go through the numerical calculations for all different parameters, since the occurrence of overstabilities is clearly understood by looking at the different frequency ranges. Using this approach we can calculate the velocity shears at which instability will occur very easily for a wide variety of parameters (for body modes at least).

For $V=0$ the first fast mode is found with a frequency around the internal Alfveń frequency. When the velocity shear is increased the frequency is dragged by the flow. It can be seen from Fig. 8 that the internal, i.e. Doppler shifted frequency, remains approximately constant and equal to the internal Alfvén frequency. An Alfvén resonance occurs when the frequency lies inside the backward Alfvén continuum starting at $-\omega_{\mathrm{Ai}}$. It is clear now that the threshold for the Alfvén RFI of the first fast wave is approximately $V=2 V_{\mathrm{Ai}}=2 V_{\mathrm{Ae}} / \sqrt{\rho_{\mathrm{i}}}$. This explains the $1 / \sqrt{\rho}$ behaviour that was found by Andries et al. (2000). Analogously we can find the velocity ranges for which the other instabilities occur. In a low- $\beta$ configuration the ranges for which slow KHI and slow resonant instability occur are very narrow, and therefore we can make no meaningful prediction concerning the occurrence of slow KHI and slow RFI in coronal plumes. We will restrict to the calculation of the velocity ranges for fast KHI and the Alfvén RFI of slow and fast body modes. Fast KHI will set in for $|V|=V_{\mathrm{Ai}}+V_{\mathrm{Ae}}$ and $|V|=V_{\mathrm{Ci}}+V_{\mathrm{Ae}}$, for the fast and slow body modes respectively. Alfvén RFI is operative for velocity shears in the ranges $2 V_{\mathrm{Ai}}<|V|<V_{\mathrm{Ai}}+V_{\mathrm{Ae}}$ and $V_{\mathrm{Ci}}+V_{\mathrm{Ai}}<|V|<k_{z} c_{\mathrm{i}}+V_{\mathrm{Ae}}$ for the first fast mode and the slow modes respectively.

\section{Summary and conclusions}

In this manuscript we investigated the effect of the velocity shear between the coronal plume and the interplume region on the spectrum of MHD waves trapped in the plume structure. More precisely we extended the analysis by Andries et al. (2000) to $\beta \neq 0$. The plume structure is modeled by a uniform interior plume region and a uniform interplume region separated by a nonuniform layer. Due to the presence of nonuniformity modes trapped in the plume structure with characteristic frequency within the range of one of the continuous spectra (associated with the nonuniform layer) may resonantly couple to localized Alfvén and/or slow continuum modes.

To illustrate the combined effect of the velocity shear and the resonant absorption process on the spectrum of the MHD trapped modes, we considered both a 1D slab configuration and a 1D cylindrical configuration.

In the case of a true discontinuity we showed that KHI occurs when the oscillation frequencies are forced into the external cut-off regions by the Doppler shift. We distinguish two types of KHI, fast and slow KHI, corresponding to the characteristics of the external waves.

In the case of a nonuniform layer, we showed that when the oscillation frequency of the forward (backward) propagating body modes are in the continuum of the forward (backward) propagating Alfvén or cusp modes, the body modes are damped due to the resonant wave excitation. However, when the oscillation frequency of the forward propagating body mode lies in the backward continua, which is only possible due to the presence of the velocity shear, the mode is overstable, i.e. the mode gains energy from the flow.

By means of the analytical dispersion relation for the true discontinuity it can clearly be seen that the internal frequency of the body modes remains more or less constant when the velocity shear changes. Using the formula of the Doppler shift between the internal and external frequency along with the external frequency ranges for which overstability will be present, we were able to determine the different unstable velocity ranges in terms of the 
characteristic velocities. This explains the dependence on the density contrast that was found in Andries et al. (2000)

As well RFI as KHI are explained by the coupling of outward to inward flux at the discontinuous flow boundary if the velocity shear is high enough. This treatment is equivalent to the negative energy wave picture used before by Cairns (1979) and by Ryutova (1988) but emphasizes more clearly the localization of the energy transfer at the flow boundary and not at the resonance layer. However this suggests that there might be an important effect of the flow profile since it would not only Doppler shift the continua but would also affect the energy exchange. This makes the conclusions for plumes somewhat uncertain, but on the other hand this suggests that some profiles are more likely to occur than others because they are more stable. We plan to investigate this further.

The most important feature of RFI is that its threshold velocity (in our model) is smaller than the threshold velocity for the onset of KHI. Since the Alfvén speed drops with distance from the sun, while the velocity shear increases (Suess 1998), the lower the velocity shear threshold the closer to the sun the instability will set in. Therefore the present results seem to suggest that Alfvén RFI of slow waves will be the most probable mixing mechanism of plume and interplume plasma. However we must keep in mind the unknown influence of the velocity profile which is likely to effect RFI more than KHI. Both mechanisms also incorporate the direct generation of Alfvén and slow waves observed in the solar wind.

\section{References}

Appert, K., Gruber, R., \& Vaclavik, J. 1974, Phys. Fluids, 17, 1471

Andries, J., Tirry, W. J., \& Goossens, M. 2000, ApJ, 531, 561

Bodo, G., Rosner, R., Ferrari, A, \& Knobloche, E. 1989, ApJ, 342,631

Bodo, G., Rosner, R., Ferrari, A, \& Knobloche, E. 1996, ApJ, 470,797
Cairns, R. A. 1979, J. Fluid Mech., 92(1), 1

Corti, G., Poletto, G., Romoli, M., Kohl, J., \& Noci, G. 1997, in The corona and solar wind near minimum activity, 5th SOHO workshop, ed. B. Fleck, ESA SP-404, 289

Csík, Á. T., Čadež, V. M., \& Goossens, M. 1998, 339, 215

Deforest, C. E., Hoeksema, J. T., Gurman, J. B., et al. 1997, SPh, 175(2), 393

Erdélyi, R., Goossens, M., \& Ruderman, M. S. 1995, SPh, 161, 123

Edwin, P. M., \& Roberts, B. 1982, SPh, 76, 239

Edwin, P. M., \& Roberts, B. 1983, SPh, 88, 179

Goossens, M., Ruderman, M. S., \& Hollweg, J. V. 1995, SPh, 157,75

Grall, R. R., Coles, W. A., Klinglesmith, M. T., et al. 1996, Nature, 379, 429

Habbal, S. R., Esser, R., Guhathakurta, M., \& Fisher, R. R. 1995, Geophys. Res. Lett., 22, 1465

Hardee, P. E., \& Clarke, D. A. 1995, ApJ, 451, L25

Hollweg, J. V., Yang, G., Čadež, V. M., \& Gaković, B. 1990, ApJ, 349, 335

Nakariakov, V. M., \& Roberts, B. 1995, SPh, 159, 213

Nakariakov, V. M., Roberts, B., \& Mann, G. 1996, A\&A, 311, 311

Parhi, S., Suess, S. T., \& Sulkanen, M. 1999, J. Geophys. Res., 104, 14, 781

Phillips, J. L., Bame, S. J., Barnes, A., et al. 1995, Geophys. Res. Lett., 22, 3301

Poletto, G., Corti, G., Kohl, J., Noci, G., \& Suess, S. T. 1997, BAAS, 29(2), 880

Ruderman, M. S., \& Goossens, M. 1995, J. Plasma Phys., $54(2), 149$

Ryutova, M. P. 1988, J. Exper. Theoret. Phys., 94, 138

Sakurai, T., Goossens, M., \& Hollweg, J. V. 1991, SPh, 133, 227

Suess, S. T. 1998, in Solar Jets and Coronal Plumes, ESTEC, The Netherlands, ESA SP-421, 223

Tirry, W. J., \& Goossens, M. 1996, ApJ, 471, 501

Tirry, W. J., Čadež, V. M., Erdélyi, R., \& Goossens, M. 1998, A\&A, 332, 786

Walker, A. D. M. 2000, J. Plasma Phys., 63(3), 203

Wang, Y.-M. 1994, ApJ, 435, L153 